\title{
Nuclear Magnetic Field in Solids Detected with Negative-Muon Spin Rotation and Relaxation
}

\author{
Jun Sugiyama, ${ }^{1,}$ Izumi Umegaki, ${ }^{1}$ Hiroshi Nozaki, ${ }^{1}$ Wataru Higemoto, ${ }^{2,3}$ Koji Hamada, ${ }^{4}$ Soshi Takeshita, ${ }^{4}$ \\ Akihiro Koda, ${ }^{4}$ Koichiro Shimomura, ${ }^{4}$ Kazuhiko Ninomiya, ${ }^{5}$ and M. Kenya Kubo ${ }^{6}$ \\ ${ }^{1}$ Toyota Central Research \& Development Laboratories Inc., Nagakute, Aichi 480-1192, Japan \\ ${ }^{2}$ Advanced Science Research Center, Japan Atomic Energy Agency, Tokai, Ibaraki 319-1195, Japan \\ ${ }^{3}$ Department of Physics, Tokyo Institute of Technology, Meguro, Tokyo 152-8551, Japan \\ ${ }^{4}$ High Energy Accelerator Research Organization (KEK), Tokai, Ibaraki 319-1106, Japan \\ ${ }^{5}$ Department of Chemistry, Graduate School of Science, Osaka University, Machikane, Osaka 560-0054, Japan \\ ${ }^{6}$ Department of Natural Sciences, College of Liberal Arts, International Christian University, Mitaka, Tokyo 181-8585, Japan
}

(Received 9 March 2018; revised manuscript received 8 May 2018; published 20 August 2018)

\begin{abstract}
Using an intense negative muon $\left(\mu^{-}\right)$source, we have studied the internal magnetic fields in a powder sample of magnesium hydride $\left(\mathrm{MgH}_{2}\right)$. By extracting the signal from the $\mu^{-}$captured on $\mathrm{Mg}$ nuclei, we found that the negative muon spin rotation and relaxation $\left(\mu^{-} \mathrm{SR}\right)$ spectra clearly showed a Kubo-Toyabetype relaxation, which indicates a random magnetic field at the $\mathrm{Mg}$ site. The field distribution width obtained is very consistent with the predicted value at the $\mathrm{Mg}$ site estimated by dipole field calculations, supporting our claim to have observed the nuclear magnetic fields of hydrogens in $\mathrm{MgH}_{2}$. As is the case with $\mu^{+} \mathrm{SR}, \mu^{-} \mathrm{SR}$ promises to soon be an indispensable tool for materials analyses.
\end{abstract}

DOI: 10.1103/PhysRevLett.121.087202

Positive muon spin rotation, relaxation, and resonance $\left(\mu^{+} \mathrm{SR}\right)$ is a technique commonly used to investigate internal magnetic fields $\left(H_{\text {int }}\right)$ in solids, due to its unique time window and time resolution [1,2]. By contrast, $\mu^{-} \mathrm{SR}$ (the negative muon counterpart of $\mu^{+} \mathrm{SR}$ ) has seen only limited use for detecting $H_{\text {int }}$ [3-8]. This is partly because at least $3 / 4$ of the $\mu^{-}$spin polarization is lost during the cascade of the $\mu^{-}$from the outermost shell orbit to the inner orbits of a muonic atom, whereas the $\mu^{+}$stops almost $100 \%$ spin polarized at the interstitial site in the lattice. This means that $\mu^{-} \mathrm{SR}$ measurements require very high statistics to obtain reliable data compared with $\mu^{+} \mathrm{SR}$, making it difficult to complete an experiment within limited beam time. Another problem is that the $\mu^{-} \mathrm{SR}$ signal is only "simple" in muonic atoms with zero nuclear spin; in others, there is an enormous hyperfine interaction locking the spin of the nucleus to that of the muon $[9,10]$. A further complication arises from the widely differing lifetimes of the $\mu^{-}$in samples composed of several different elements. This problem is largely avoided in magnesium hydride $\left(\mathrm{MgH}_{2}\right)$ because those muons that are initially captured on hydrogen very quickly transfer to $\mathrm{Mg}$ nuclei [11] — but at the expense of a further polarization loss in the muonic hydrogen stage.

A very recent development in the $\mu^{-}$beam and counting system in Japan Proton Accelerator Research Complex (J-PARC) [12] has drastically increased the counting rate of $\mu^{-}$SR to over $2 \times 10^{4}$ events/s ( $72 \mathrm{M}$ events/hr), allowing completion of a $\mu^{-} \mathrm{SR}$ experiment in an acceptable period. Here we report the first observation with $\mu^{-} \mathrm{SR}$ of the nuclear magnetic field in $\mathrm{MgH}_{2}$ [see Fig. 1(a)].
Our original motivation for studying $\mathrm{MgH}_{2}$ with muons was to elucidate the predominant parameter determining the hydrogen desorption temperature $\left(T_{d}\right)$ [15-18]. In fact, with $\mu^{+} \mathrm{SR}$ we found that liberated hydrogen starts to diffuse below $T_{d}$ in milled $\mathrm{MgH}_{2}[19,20]$. However, since $\mu^{+}$behaves like a light isotope of $\mathrm{H}^{+}$in solids, $\mu^{+}$would diffuse more rapidly than $\mathrm{H}$ in $\mathrm{MgH}_{2}$ [21,22], and either type of diffusion will have the same effect on $\mu^{+}$spin relaxation. Thus, it is difficult to estimate the diffusion coefficient of $\mathrm{H}$ with $\mu^{+} \mathrm{SR}$ without ambiguity.

The implanted $\mu^{-}$in $\mathrm{MgH}_{2}$ should be captured on $\mathrm{Mg}$, effectively converting $\mathrm{Mg}$ to $\mathrm{Na}$ chemically. Since about $90 \%$ of $\mathrm{Mg}$ nuclei lack a nuclear spin, the $\mu^{-}$spin in muonic Mg directly senses a local $H_{\text {int }}$ at the Na-like site, mainly caused by the surrounding $\mathrm{H}$ spins. When $\mathrm{H}$ starts to diffuse, $\mu^{-} \mathrm{SR}$ thus detects a dynamic nuclear magnetic field and directly reveals the hopping rate of $\mathrm{H}$ diffusion. Past $\mu^{-}$SR experiments showed that the $\mu^{-}$decay asymmetry in $\mathrm{Mg}$ metal is 0.037 [23] or 0.05 [24] -i.e., about $1 / 6-1 / 5$ of the $\mu^{+}$decay asymmetry (typically about 0.24 in usual experimental conditions). This means that we need at least 36-25 times higher statistics than that for $\mu^{+}$SR to obtain the statistical precision for a meaningful measurement of the $\mu^{-} \mathrm{SR}$ spectrum in $\mathrm{Mg}$ compounds.

A powder sample of $\mathrm{MgH}_{2}$ was purchased from Wako Pure Chemical Industries, Ltd. The $\mu^{-}$SR time spectra were measured on the decay muon beam line D1 at MUSE of MLF J-PARC in Japan. The approximately $40 \mathrm{~g}$ powder sample was placed in a plastic case with $5 \times 5 \times 2 \mathrm{~cm}^{3}$ volume, made of a $0.5 \mathrm{~mm}$-thick polyethylene terephthalate 
(PET) plate. The plastic case was then sealed in an Al-coated plastic bag in a He-filled glove box, because $\mathrm{MgH}_{2}$ is unstable in air. The sealed plastic bag was set at the center of the spectrometer. The momentum of the $\mu^{-}$ beam was adjusted to $50 \mathrm{MeV} / c$ to maximize the number of $\mu^{-}$stopped in the sample. The $\mu^{-} \mathrm{SR}$ spectrum was measured at room temperature with up to 200-300 Mevents for transverse field (TF) $\mu^{-}$SR and 500-1000 Mevents for zero field (ZF) and longitudinal field (LF) $\mu^{-} \mathrm{SR}$ at a counting rate of 72 Mevents/hr. Here TF (LF) means the field perpendicular (parallel) to the initial $\mu^{-}$spin polarization. Commercially available $\mathrm{Mg}$ metal ribbon, $\mathrm{MgF}_{2}$ and $\mathrm{MgO}$ powders, and PET plates were also measured using the same setup for comparison. The experimental techniques are described in more detail elsewhere $[1,2]$. The obtained $\mu^{-}$SR data was analyzed with MUSRFIT [25].

To demonstrate the characteristic features of the $\mu^{-} \mathrm{SR}$ spectra, Figs. 1(b) and 1(c) show the time histograms of the forward and backward counters $\left[N^{F}(t)\right.$ and $\left.N^{B}(t)\right]$ recorded in a TF with $H=50$ Oe. Here, forward (backward) means upstream (downstream) of the sample in the $\mu^{-}$beam. Since the decay asymmetry is very small and the

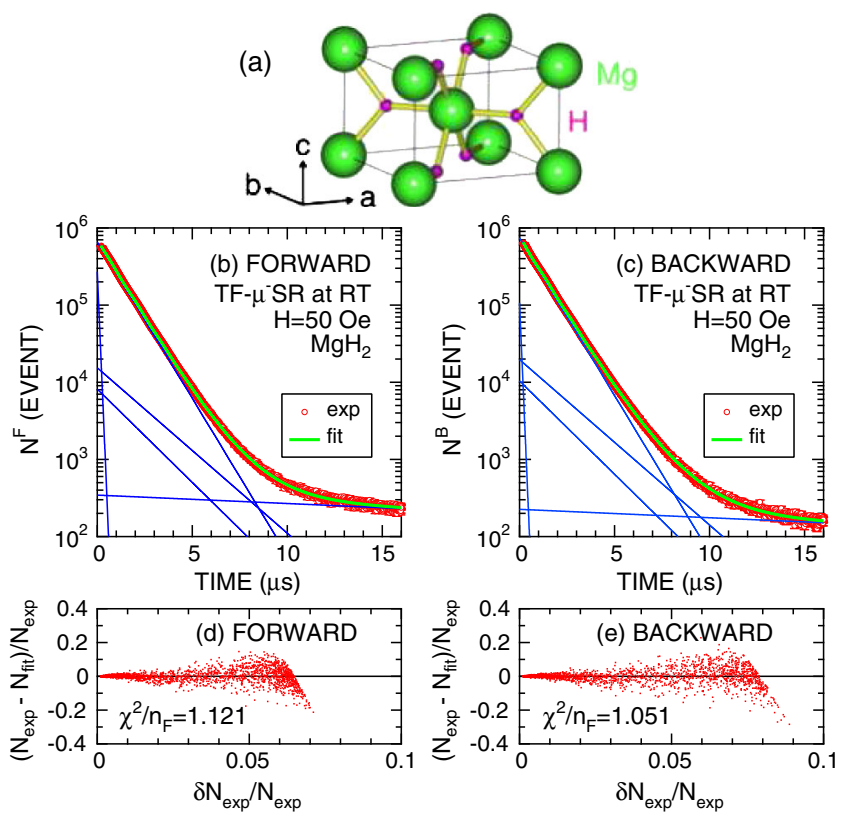

FIG. 1. (a) The crystal structure of tetragonal $\mathrm{MgH}_{2}$ drawn with VESTA [13]. The lattice constants are $a=4.5180(6) \AA$ and $c=$ 3.0211(4) $\AA$ with space group $P 4_{2} / m n m$ [14]. The time histogram of the $\mathrm{TF}-\mu^{-} \mathrm{SR}$ spectrum in $\mathrm{MgH}_{2}$ for the (b) forward counter and (c) backward counter, and (d) and (e) the reduced difference between the experimental data and fit result $\left(\left[N_{\exp }-N_{\text {fit }}\right] /\left[N_{\text {exp }}\right]\right)$ as a function of the reduced experimental error $\left(\delta N_{\exp } / N_{\text {exp }}\right)$. In (b) and (c), red open circles represent the experimental data, green solid lines represent the fit result using Eq. (1), and blue solid lines represent the histograms of the five decay processes (see Table I). In (d) and (e), since the fit was performed to minimize $\chi^{2}$, reduced $\chi^{2}\left(=\chi^{2} / n_{F}\right)$ for the data is also shown, where $n_{F}$ is the number of degree of freedom. lifetime of the $\mu^{-}$depends upon the nucleus on which it captures, the histogram was fitted by a combination of five different decay processes:

$$
N(t)=\sum_{i=1}^{5} N_{i} e^{-t / \tau_{i}}\left[1+A_{i} e^{-\lambda_{i} t} \cos \left(\omega_{i} t+\phi_{i}\right)\right],
$$

where $N_{i}$ is a normalization constant at $t=0$ for the $i$ th decay process, $\tau_{i}$ is the corresponding muon lifetime for that process, $A_{i}$ is the average muon decay asymmetry for that process, $\omega_{i}$ is the angular frequency of the $\mu^{-}$spin precession caused by the applied TF, and $\phi_{i}$ is the initial phase. $A_{i}$ is nonzero only for the predominant process and zero for the other minor processes. The timing that $t=0$ for each histogram was determined so as to $\phi^{F}=-180^{\circ}$ and $\phi^{B}=0^{\circ}$, because the initial $\mu^{-}$spin polarization is parallel to its momentum.

The fit showed that the first decay process with $\tau_{\mathrm{Mg}}=$ $1.067 \mu \mathrm{s}$ is predominant in the time domain between 0.2 and $\sim 8 \mu \mathrm{s}$, where $\tau_{\mathrm{Mg}}$ means $\tau$ for $\mu^{-}$captured on ${ }^{24} \mathrm{Mg}$ [26]. The second and third decay processes represent muons captured on ${ }^{12} \mathrm{C}$ with $\tau_{\mathrm{C}}=2.0263 \mu$ s and ${ }^{16} \mathrm{O}$ with $\tau_{\mathrm{O}}=1.795 \mu \mathrm{s}$ [26] in the sample case made of PET. Here, we fixed the ratio $N_{3} / N_{2}$ at 0.53 from the result on PET (see Table I).

The fourth decay process represents $\mu^{-}$captured on $\mathrm{Pb}$ with $\tau_{\mathrm{Pb}}=0.0754 \mu \mathrm{s}$ [26], because the spot size of the $\mu^{-}$ beam was adjusted using a $\mathrm{Pb}$ collimator. This is consistent with the result that $N_{4}^{F} / N_{1}^{F}>N_{4}^{B} / N_{1}^{B}$. The fact that $\tau_{5}$ is longer than $\tau$ for a free $\mu^{ \pm}(2.196 \mu \mathrm{s})$ shows that the fifth decay process is caused by other particles, such as $e^{-}, e^{+}$, and/or neutrons, or the other effects for reasons currently unknown. However, the contribution of the fifth decay process is negligibly small at $t<\sim 6 \mu \mathrm{s}$.

Figure 2 shows the two TF- $\mu^{-} \mathrm{SR}$ asymmetry spectra $[1,2,25]$ measured with $H=50$ and 130 Oe. The contributions from the second to fifth decay processes are different in the forward counter from those in the backward counter (see Fig. 1). This leads to a nonlinear background (BG), particularly at $t \geq 6 \mu \mathrm{s}$, where such contributions dominate those from the decay of $\mu^{-}$captured on $\mathrm{Mg}$. However, this nonlinear BG is found to be independent of the external field [Fig. 2(b)]. Considering the distortion of the spectrum at early times, we attempted to fit the $\mu^{-} \mathrm{SR}$ asymmetry spectra in the time domain between 0.4 and $5.5 \mu$ s together with subtracting the nonlinear $\mathrm{BG}$, as a first approximation.

Figure 3 shows the TF-, ZF-, and three LF- $\mu^{-}$SR asymmetry spectra for $\mathrm{MgH}_{2}$. The ZF and LF spectra were fitted by a combination of a dynamic Gaussian Kubo-Toyabe function $\left(G^{\mathrm{DGKT}}\right)$ [27] and an exponential relaxation function:

$$
A_{0} P(t)=A_{\mathrm{KT}} G^{\mathrm{DGKT}}\left(t, \Delta, \nu, H_{\mathrm{LF}}\right)+A_{T} e^{-\lambda_{T} t},
$$

where $A_{0}$ is the initial ( $\left.t=0\right)$ asymmetry, $A_{\mathrm{KT}}$ and $A_{T}$ are the asymmetries associated with the signals from the $\mu^{-}$, which is captured on $\mathrm{Mg}$, feeling a nuclear field and a fluctuating 
TABLE I. Parameters of the TF- $\mu^{-} \mathrm{SR}$ histograms for $\mathrm{Mg}, \mathrm{MgH}_{2}, \mathrm{MgF}_{2}$, and $\mathrm{MgO}$ samples and PET plates obtained by fitting with Eq. (1), in which the number of decay processes is 5 for $\mathrm{Mg}, \mathrm{MgH}_{2}$, and $\mathrm{MgO}, 6$ for $\mathrm{MgF}_{2}$, and 4 for PET. Here, $\tau_{\mathrm{C}}=2.0263(15) \mu$ s for ${ }^{12} \mathrm{C}, \tau_{\mathrm{O}}=1.795(2) \mu \mathrm{s}$ for ${ }^{16} \mathrm{O}, \tau_{\mathrm{F}}=1.463(5) \mu \mathrm{s}$ for ${ }^{19} \mathrm{~F}, \tau_{\mathrm{Mg}}=1.067(2) \mu \mathrm{s}$ for ${ }^{24} \mathrm{Mg}$, and $\tau_{\mathrm{Pb}}=0.0754(10) \mu \mathrm{s}$ for ${ }^{82} \mathrm{~Pb}$ [26]. Since it is extremely difficult to determine a short $\tau$ using data at early times due to the effect of beam $e^{ \pm}$hitting the counters directly, we fitted the histograms only in the time domain between 0.2 and $16 \mu \mathrm{s}$. Note that the ratio between the major components for the forward counter is very close to that for the backward counter; that is, $N_{F}^{F} / N_{\mathrm{Mg}}^{F} \sim N_{F}^{B} / N_{\mathrm{Mg}}^{B} \sim 2.26$ in $\mathrm{MgF}_{2}, N_{\mathrm{O}}^{F} / N_{\mathrm{Mg}}^{F} \sim N_{\mathrm{O}}^{B} / N_{\mathrm{Mg}}^{B} \sim 1.15$ in $\mathrm{MgO}$, and $N_{\mathrm{O}}^{F} / N_{\mathrm{C}}^{F} \sim N_{\mathrm{O}}^{B} / N_{\mathrm{C}}^{B} \sim 0.53$ in PET. The histograms for $\mathrm{Mg}, \mathrm{MgH}_{2}$, and $\mathrm{MgF}_{2}$ were fitted using a common $N_{\mathrm{O}} / N_{\mathrm{C}}$, i.e., $N_{\mathrm{O}}^{F} / N_{\mathrm{C}}^{F}=N_{\mathrm{O}}^{B} / N_{\mathrm{C}}^{B}=0.53$, because such components come from the PET sample case.

\begin{tabular}{|c|c|c|c|c|c|c|c|}
\hline Material & $\tau_{i}(\mu \mathrm{s})$ & $N_{i}^{F} / N_{1}^{F}$ & $N_{i}^{B} / N_{1}^{B}$ & $A_{i}^{F}$ & $A_{i}^{B}$ & $\lambda_{i}^{F}\left(\mu \mathrm{s}^{-1}\right)$ & $\lambda_{i}^{B}\left(\mu \mathrm{s}^{-1}\right)$ \\
\hline $\mathrm{Mg}$ & $\begin{array}{l}\tau_{1}=\tau_{\mathrm{Mg}} \\
\tau_{2}=\tau_{\mathrm{C}} \\
\tau_{3}=\tau_{\mathrm{O}} \\
\tau_{4}=\tau_{\mathrm{Pb}} \\
\tau_{5}=36.7(1.3)\end{array}$ & $\begin{array}{l}1 \\
0.01747(9) \\
0.0093 \\
0.353(9) \\
0.000547(7)\end{array}$ & $\begin{array}{l}1 \\
0.02081(7) \\
0.0110 \\
0.202(9) \\
0.000304(4)\end{array}$ & $\begin{array}{l}0.0356(2) \\
\ldots \\
\ldots \\
\ldots \\
\ldots\end{array}$ & $\begin{array}{l}0.03371(19) \\
\ldots \\
\ldots \\
\ldots \\
\ldots\end{array}$ & $0.0000(12)$ & $0.0000(15)$ \\
\hline $\mathrm{MgH}_{2}$ & $\begin{array}{l}\tau_{1}=\tau_{\mathrm{Mg}} \\
\tau_{2}=\tau_{\mathrm{C}} \\
\tau_{3}=\tau_{\mathrm{O}} \\
\tau_{4}=\tau_{\mathrm{Pb}} \\
\tau_{5}=20.0(5)\end{array}$ & $\begin{array}{l}1 \\
0.0229(9) \\
0.012 \\
0.404(12) \\
0.00051(7)\end{array}$ & $\begin{array}{l}1 \\
0.02690(7) \\
0.014 \\
0.147(12) \\
0.00031(4)\end{array}$ & $\begin{array}{l}0.0196(5) \\
\ldots \\
\ldots \\
\ldots \\
\ldots\end{array}$ & $\begin{array}{l}0.0213(6) \\
\ldots \\
\ldots \\
\ldots \\
\ldots\end{array}$ & $0.22(2)$ & $0.32(2)$ \\
\hline $\mathrm{MgF}_{2}$ & $\begin{array}{l}\tau_{1}=\tau_{\mathrm{Mg}} \\
\tau_{2}=\tau_{\mathrm{C}} \\
\tau_{3}=\tau_{\mathrm{O}} \\
\tau_{4}=\tau_{F} \\
\tau_{5}=\tau_{\mathrm{Pb}} \\
\tau_{6}=24.3(1.1)\end{array}$ & $\begin{array}{l}1 \\
0.0304(18) \\
0.016 \\
2.216(16) \\
0.81(3) \\
0.00185(6)\end{array}$ & $\begin{array}{l}1 \\
0.0437(18) \\
0.023 \\
2.301(17) \\
0.77(3) \\
0.00153(5)\end{array}$ & $\begin{array}{l}0.0040(3)^{\mathrm{a}} \\
\ldots \\
\ldots \\
\ldots \\
\ldots \\
\ldots\end{array}$ & $\begin{array}{l}0.0040(3)^{\mathrm{a}} \\
\ldots \\
\ldots \\
\ldots \\
\ldots \\
\ldots\end{array}$ & $0.25(5)^{\mathrm{a}}$ & $0.25(5)^{\mathrm{a}}$ \\
\hline $\mathrm{MgO}$ & $\begin{array}{l}\tau_{1}=\tau_{\mathrm{Mg}} \\
\tau_{2}=\tau_{\mathrm{C}} \\
\tau_{3}=\tau_{\mathrm{O}} \\
\tau_{4}=\tau_{\mathrm{Pb}} \\
\tau_{5}=20(3)\end{array}$ & $\begin{array}{l}1 \\
0.092(18) \\
1.13(2) \\
1.08(6) \\
0.0018(2)\end{array}$ & $\begin{array}{l}1 \\
0.102(12) \\
1.166(16) \\
0.76(5) \\
0.00096(12)\end{array}$ & $\begin{array}{l}0.0035(6)^{\mathrm{a}} \\
\ldots \\
\ldots \\
\ldots \\
\ldots\end{array}$ & $\begin{array}{l}0.0035(6)^{\mathrm{a}} \\
\ldots \\
\ldots \\
\ldots \\
\ldots\end{array}$ & $0.24(12)^{\mathrm{a}}$ & $0.24(12)^{\mathrm{a}}$ \\
\hline PET & $\begin{array}{l}\tau_{1}=\tau_{\mathrm{C}} \\
\tau_{2}=\tau_{\mathrm{O}} \\
\tau_{3}=\tau_{\mathrm{Pb}} \\
\tau_{4}=26.2(1.8)\end{array}$ & $\begin{array}{l}1 \\
0.532(2) \\
0.467(17) \\
0.00111(4) \\
\end{array}$ & $\begin{array}{l}1 \\
0.5299(17) \\
0.372(17) \\
0.00055(2) \\
\end{array}$ & $\begin{array}{l}0.0401(6) \\
\ldots \\
\ldots \\
\ldots \\
\end{array}$ & $\begin{array}{l}0.0369(6) \\
\ldots \\
\ldots \\
\ldots \\
\end{array}$ & $0.320(10)$ & $0.291(9)$ \\
\hline
\end{tabular}

${ }^{\mathrm{a}}$ Due to a very small asymmetry, $A$ and $\lambda$ were obtained by fitting the TF-asymmetry spectrum with $A e^{-\lambda t} \cos (\omega t+\phi)$.

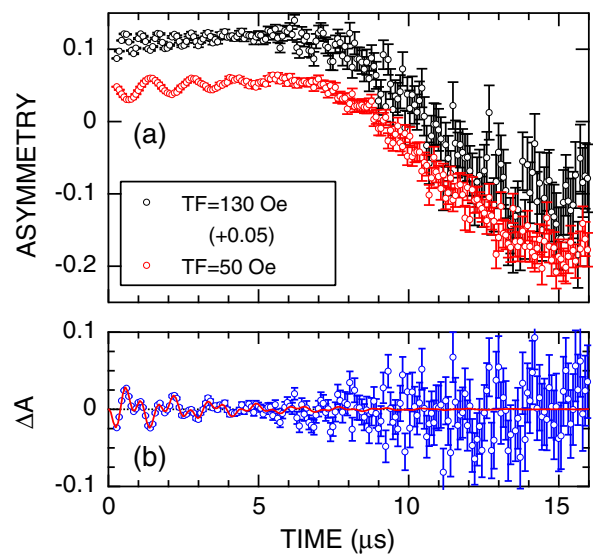

FIG. 2. (a) The $\mathrm{TF}-\mu^{-} \mathrm{SR}$ asymmetry spectra for $\mathrm{MgH}_{2}$ recorded with $H=50$ and 130 Oe. (b) The difference between the above two asymmetry spectra. In (a), the TF spectrum with 130 Oe is shifted upward by 0.05 for clarity of display. In (b), a solid line represents the fit using a combination of two cosine functions due to the TF oscillations with $H=50$ and 130 Oe. magnetic field, respectively, $\Delta$ is the static width of the local field distribution at the disordered sites, $\nu$ is the fluctuation rate of the fields, and $\lambda_{T}$ is the exponential relaxation rate. $\Delta[\nu]$ corresponds to a spin-spin relaxation rate $\left(1 / T_{2}\right)$ [a spin-lattice relaxation rate $\left.\left(1 / T_{1}\right)\right][1,2]$. For $\nu=0$ and $H_{\mathrm{LF}}=0$ (i.e., $\left.\mathrm{ZF}\right), G^{\mathrm{DGKT}}\left(t, \Delta, \nu, H_{\mathrm{LF}}\right)$ becomes a static Gaussian Kubo-Toyabe function [28], $G_{z z}^{\mathrm{KT}}(t, \Delta)=$ $\frac{1}{3}+\frac{2}{3}\left(1-\Delta^{2} t^{2}\right) \exp \left(-\frac{1}{2} \Delta^{2} t^{2}\right)$.

Using common $A_{\mathrm{KT}}, A_{T}, \nu, \Delta$, and $\lambda_{T}$ for the $\mathrm{ZF}$ and three LF spectra, the fit yielded $A_{\mathrm{KT}}=0.0123(4)$, $A_{T}=0.0094(4), \Delta=0.520(7) \mu \mathrm{s}^{-1}$ [equivalent to a field distribution width of 6.11(8) Oe], $\nu=0.10(3) \mu \mathrm{s}^{-1}$, and $\lambda_{T}=0.40(2) \mu \mathrm{s}^{-1}$. Dipole field calculations with DiPELEC [29] predict $\Delta^{\text {calc }}=0.5807 \mu \mathrm{s}^{-1}[6.819$ Oe] at the Mg site, consistent with the fitted $\Delta$ from $\mu^{-}$SR. Hence, it is clarified that $\mu^{-}$feels the $H_{\text {int }}$ at $\mathrm{Mg}$. Since $\nu$ is about $1 / 5$ of $\Delta, H_{\text {int }}$ is fluctuating, probably due to thermal vibration or hopping of $\mathrm{H}$, as proposed by proton nuclear magnetic resonance ( ${ }^{1} \mathrm{H}-\mathrm{NMR}$ ) [30]. The $A_{T}$ signal is likely caused by free 


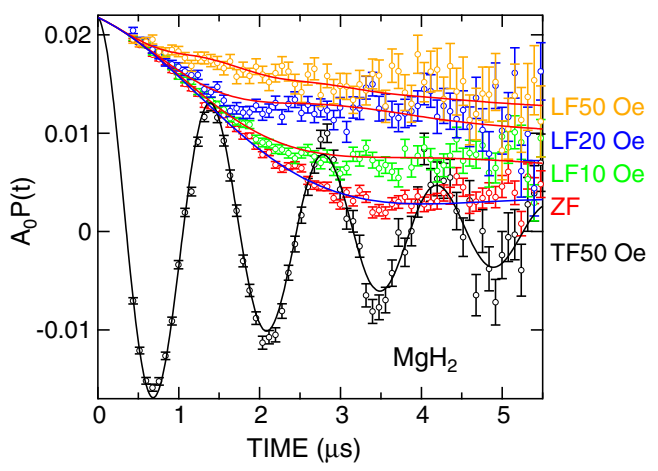

FIG. 3. The TF-, ZF-, and LF- $\mu^{-}$SR asymmetry spectra for $\mathrm{MgH}_{2}$ recorded at room temperature. The TF spectrum was fitted by an exponentially relaxing cosine signal: $A_{0} P_{\mathrm{TF}}(t)=$ $A_{\mathrm{TF}} \exp \left(-\lambda_{\mathrm{TF}} t\right) \cos \left(\omega_{\mathrm{TF}} t+\phi\right)$ with $A_{\mathrm{TF}}=0.02177(14), \quad \phi=$ $-0.4(1.0) \mathrm{deg}$, and $\lambda_{\mathrm{TF}}=0.365(9) \mu \mathrm{s}^{-1}$, which corresponds to $1 / T_{2}$ as well as $\Delta\left[=0.545(9) \mu \mathrm{s}^{-1}\right]$. It is known that $\lambda_{\mathrm{TF}}<$ $\Delta$ [19], being consistent with the present result.

electrons or other paramagnetic species, which are created with the cascade process of the $\mu^{-}$captured on $\mathrm{Mg}$ and/or the transfer of the $\mu^{-}$captured on $\mathrm{H}$ to $\mathrm{Mg}$ nuclei [11]. The $\mu^{-}$on $\mathrm{Mg}$ being close to such species feels a large fluctuating magnetic field, which hides a small nuclear magnetic field.

The muonic $\mathrm{Mg}$, which behaves as a Na-like atom, naturally perturbs local structural environments. However, it is very hard to estimate correct $\Delta^{\text {calc }}$ due to the absence of $\mathrm{NaH}_{2}$. Considering the Na-H bond length $\left(d_{\mathrm{Na}-\mathrm{H}}=2.49 \AA\right)$ in $\mathrm{NaH}[31], d_{\mathrm{Na}-\mathrm{H}}$ is expected to be longer than $d_{\mathrm{Mg}-\mathrm{H}}$ (= 1.93 or $1.97 \AA$ ) in $\mathrm{MgH}_{2}$. This leads to smaller $\Delta$ at the $\mathrm{Na}$ site than that at the $\mathrm{Mg}$ site in $\mathrm{MgH}_{2}$, because $\Delta^{\text {calc }}=$ 3.318 Oe for $\mathrm{NaH}$ without a contribution from ${ }^{23} \mathrm{Na}$.

The $\mu^{-} \mathrm{s}$ captured on the $10 \%$ abundant ${ }^{25} \mathrm{Mg}$ with $I=5 / 2$ have their spins "locked" to the nuclear spin by a huge hyperfine interaction; these $\mu^{-}$s precess at a completely different frequency and have a negligible effect on the $\mu^{-} \mathrm{SR}$ spectrum in low or zero field.

Earlier $\mu^{+} \mathrm{SR}$ measurements on $\mathrm{MgH}_{2}[19,20]$ revealed that while the $\mu^{+}$is static in a 3 -spin $1 / 2$ " $\mathrm{H}-\mu$-H" system at low temperatures, by room temperature it has partially delocalized into a channel along the $c$ axis near the $\mu^{+}$site $(0.5185,0.9984,0.7162)$ predicted by first principles calculations [32], which showed that the electrostatic potential at $(0.5185,0.9984, z)$ is almost independent of $z$. Dipole field calculations also suggest that $\Delta^{\text {calc }}$ is roughly independent of $z$; i.e., $\Delta^{\text {calc }}$ ranges between 0.629 and $0.664 \mu \mathrm{s}^{-1}[7.39$ and 7.80 Oe]. This means that the delocalized $\mu^{+}$feels only the average of the static dipolar field over that region, providing $\Delta=0.332(6) \mu \mathrm{s}^{-1}$ $\left[3.90(6)\right.$ Oe], and that $\mu^{+}$diffusion then presumably involves "hopping" from one channel to an adjacent one making the $\mu^{+} \mathrm{SR}$ signal less sensitive to $\mathrm{H}$ diffusion. Furthermore, $\mu^{+}$diffusion would be correlated with $\mathrm{H}$

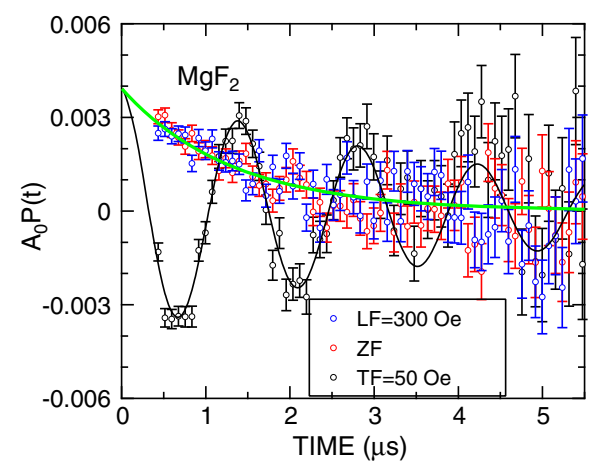

FIG. 4. The TF-, ZF-, and LF- $\mu^{-}$SR asymmetry spectra for $\mathrm{MgF}_{2}$ recorded at room temperature. The green bold line shows a best fit for the $\mathrm{ZF}$ and $\mathrm{LF}$ spectra using an exponential relaxation signal: $A_{0} P(t)=A_{F} \exp \left(-\lambda_{F} t\right)$ with $\lambda_{F}=0.77(5) \mu \mathrm{s}^{-1}$. Also, $A_{\mathrm{TF}}=0.0040(3)=A_{F}, \phi=6(4) \mathrm{deg}$, and $\lambda_{\mathrm{TF}}=0.25(5) \mu \mathrm{s}^{-1}$ (see Table I and the caption of Fig. 3).

diffusion. This may explain why the measured value of $\nu=0.045(10) \mu \mathrm{s}^{-1}$ for $\mu^{+} \mathrm{SR}$ at $300 \mathrm{~K}$ is less than that for $\mu^{-} \mathrm{SR}$, whose lack of ambiguity illustrates the superior power of the latter for measuring dynamic $H_{\text {int }}$ in solids.

Figure 4 shows the TF-, ZF-, and LF- $\mu^{-}$SR spectra for $\mathrm{MgF}_{2}$. Despite the same structure (with space group $\mathrm{P4}_{2} / \mathrm{mnm}$ ) of $\mathrm{MgF}_{2}$ [33] as that of $\mathrm{MgH}_{2}$, the $\mathrm{ZF}$ spectrum is very different from that for $\mathrm{MgH}_{2}$ (see Fig. 3); i.e., it exhibits an exponential relaxation behavior. Here, we should note that the natural abundance of ${ }^{19} \mathrm{~F}$ with $I=1 / 2$ is $100 \%$ and the capture ratio between $\mathrm{Mg}$ and $\mathrm{F}$ is $1.13\left(=\frac{2.26}{2}\right)$ (Table I), being comparable to the reported value (0.92) [34]. This means that a majority of $\mu^{-}$is captured on $\mathrm{F}$ and, as a result, such $\mu^{-}$spin is "locked" to the nuclear spin of $\mathrm{F}$.

In summary, we have observed a Kubo-Toyabe relaxation due to the nuclear spin of $\mathrm{H}$ in $\mathrm{MgH}_{2}$ with a negative muon spin rotation and relaxation $\left(\mu^{-} \mathrm{SR}\right)$ technique. This will open the door to studying the dynamic behavior of light elements (ions) in solids with $\mu^{-} \mathrm{SR}$ from the fixed viewpoint of the nucleus, giving for once a clear advantage over $\mu^{+} \mathrm{SR}$. More correctly, the $\mu^{-}$captured on light elements with $I=0$, such as, ${ }^{12} \mathrm{C},{ }^{16} \mathrm{O},{ }^{24} \mathrm{Mg},{ }^{28} \mathrm{Si}, \ldots$, has a sufficiently long lifetime for measuring a dynamic behavior of surrounding atoms and/or ions with $I \geq \frac{1}{2}$. Hence, $\mu^{-}$SR is a complementary technique to NMR and a useful tool for energy materials research.

We thank the staff of J-PARC (especially the MUSE) for help with the $\mu^{-} \mathrm{SR}$ experiments (Proposals No. 2017B0137 and No. 2018A0090) and J. H. Brewer for discussion and editing. This work was supported by Japan Society for the Promotion Science (JSPS) KAKENHI Grants No. JP26286084 and No. JP18H01863.

*e0589@mosk.tytlabs.co.jp; juns@triumf.ca

[1] G. M. Kalvius, D. R. Noakes, and O. Hartmann, Handbook on the Physics and Chemistry of Rare Earths (North-Holland, Amsterdam, 2001), Vol. 32, Chap. 206, pp. 55-451. 
[2] A. Yaouanc and P.D. de Réotier, Muon Spin Rotation, Relaxation, and Resonance, Application to Condensed Matter (Oxford, New York, 2011).

[3] S. Nagamiya, K. Nagamine, O. Hashimoto, and T. Yamazaki, Phys. Rev. Lett. 35, 308 (1975).

[4] K. Nagamine, S. Nagamiya, O. Hashimoto, S. Kobayashi, and T. Yamazaki, Hyperfine Interact. 2, 407 (1976).

[5] J. Imazato, K. Nagamine, T. Yamazaki, B. D. Patterson, E. Holzschuh, and R. F. Kiefl, Phys. Rev. Lett. 53, 1849 (1984).

[6] R. Kadono, J. Imazato, T. Ishikawa, K. Nishiyama, K. Nagamine, T. Yamazaki, A. Bosshard, M. Döbeli, L. van Elmbt, M. Schaad, P. Truöl, A. Bay, J. P. Perroud, J. Deutsch, B. Tasiaux, and E. Hagn, Phys. Rev. Lett. 57, 1847 (1986).

[7] H. Keller, R. F. Kiefl, H. Baumeler, W. Kündig, B. D. Patterson, J. Imazato, K. Nishiyama, K. Nagamine, T. Yamazaki, and R. I. Grynszpan, Phys. Rev. B 35, 2008 (1987).

[8] N. Nishida, Hyperfine Interact. 79, 823 (1993).

[9] J. H. Brewer, Hyperfine Interact. 19, 873 (1984).

[10] J. H. Brewer, K. Ghandi, A. M. Froese, and B. A. Fryer, Phys. Rev. C 71, 058501 (2005).

[11] L. I. Ponomarev, Annu. Rev. Nucl. Sci. 23, 395 (1973).

[12] K. M. Kojima et al., J. Phys. Conf. Ser. 551, 012063 (2014).

[13] K. Momma and F. Izumi, J. Appl. Crystallogr. 41, 653 (2008).

[14] T. Noritake, M. Aoki, S. Towata, Y. Seno, Y. Hirose, E. Nishibori, M. Takata, and M. Sakata, Appl. Phys. Lett. 81, 2008 (2002).

[15] G. Liang, J. Huot, S. Boily, A. V. Neste, and R. Schulz, J. Alloys Compd. 292, 247 (1999).

[16] J.-L. Bobet, B. Chevalier, M. Song, B. Darriet, and J. Etourneau, J. Alloys Compd. 336, 292 (2002).

[17] N. Hanada, T. Ichikawa, and H. Fujii, J. Alloys Compd. 446-447, 67 (2007).
[18] J. Sugiyama, Y. Ikedo, T. Noritake, O. Ofer, T. Goko, M. Månsson, K. Miwa, E. J. Ansaldo, J. H. Brewer, K. H. Chow, and S.-i. Towata, Phys. Rev. B 81, 092103 (2010).

[19] I. Umegaki, H. Nozaki, M. Harada, Y. Higuchi, T. Noritake, M. Matsumoto, S.-i. Towata, E. J. Ansaldo, J. H. Brewer, A. Koda, Y. Miyake, and J. Sugiyama, J. Phys. Conf. Ser. 551, 012036 (2014).

[20] J. Sugiyama, J. Phys. Soc. Jpn. 85, 091012 (2016).

[21] J. F. Pelletier, J. Huot, M. Sutton, R. Schulz, A. R. Sandy, L. B. Lurio, and S. G. J. Mochrie, Phys. Rev. B 63, 052103 (2001).

[22] X. Yao, Z. H. Zhu, H. M. Cheng, and G. Q. Lu, J. Mater. Res. 23, 336 (2008).

[23] D. C. Buckle, J. R. Kane, R. T. Siegel, and R. J. Wetmore, Phys. Rev. Lett. 20, 705 (1968).

[24] A. Astbury, I. M. Blair, M. Hussain, M. A. R. Kemp, and H. Muirhead, Proc. Phys. Soc. 78, 1149 (1961).

[25] A. Suter and B. Wojek, Phys. Procedia 30, 69 (2012).

[26] T. Suzuki, D. F. Measday, and J. P. Roalsvig, Phys. Rev. C 35, 2212 (1987).

[27] R. S. Hayano, Y. J. Uemura, J. Imazato, N. Nishida, T. Yamazaki, and R. Kubo, Phys. Rev. B 20, 850 (1979).

[28] R. Kubo and T. Toyabe, Magnetic Resonance and Relaxation (North-Holland, Amsterdam, 1996).

[29] K. M. Kojima, J. Yamanobe, H. Eisaki, S. Uchida, Y. Fudamoto, I. M. Gat, M. I. Larkin, A. Savici, Y. J. Uemura, P. P. Kyriakou, M. T. Rovers, and G. M. Luke, Phys. Rev. B 70, 094402 (2004).

[30] Y. Itoh and R. Kado, J. Phys. Soc. Jpn. Conf. Proc. 1, 012013 (2014).

[31] C. G. Shull, E. O. Wollan, G. A. Morton, and W. L. Davidson, Phys. Rev. 73, 842 (1948).

[32] K. Miwa (private communication).

[33] G. Vidal-Valat, J.-P. Vidal, C.M.E. Zeyen, and K. Kurki-Suonio, Acta Crystallogr. Sect. B 35, 1584 (1979).

[34] W. Wilhelm, R. Bergmann, G. Fottner, F. Hartmann, J. Reidy, and H. Daniel, Chem. Phys. Lett. 55, 478 (1978). 